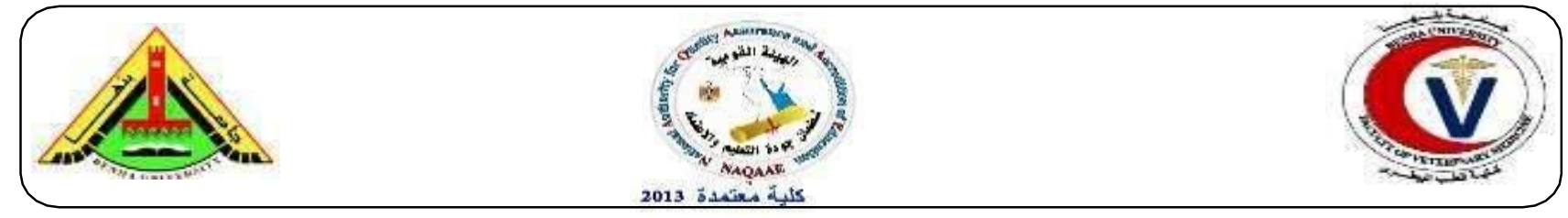

\title{
Preparation of buffered acidified plate antigen from Brucella abortus strain 19
}

${ }^{1}$ Lubna,F. Farahat, ${ }^{2}$ Khaled,A.Abd-El-Azeem, ${ }^{3}$ Alaa,El Menisy, ${ }^{4}$ Ayman, Mahrouse, ${ }^{5}$ Essam, A. Nasr.

1,2,3,5VSVRI. Abbasia, Cairo. ${ }^{4}$ Department of Public Health and Zoonoses, General Organization for Veterinary Services.

\section{A B S T R A C T}

Brucellosis is one of the major zoonotic infections worldwide, continues to be a public health problem resulting in significant morbidity and economic losses. Accurate diagnosis must include laboratory tests that allow the direct Brucella isolation or indirect detection of antibodies. Practical serological tests are routinely used for the diagnosis of brucellosis by using specific antigens. In the present work, the used buffered acidified plate antigen (BAPA) was prepared from B. abortus biovar 1 strain S1119-3 according to the USDA SOPs. For this purpose, a total of 4100 bovine sera from five farms located in different governorates were screened for brucellosis. The effectiveness of prepared BAPA from strain 19 was compared with the standard BAPA and BCT antigens prepared from B. abortus strain 99. Evaluation was done by using a panel of known dilutions of the OIEISS (Office International des Epizootie International Standard Serum) as the international reference standard serum. There were no significant differences in results of the BAPA antigen prepared from strain 19 and the conventional antigen prepared from strain 99. The results concluded that, the vaccinal strain 19 can be used instead of strain 99 to prepare the BAPA antigen.

Key word: Bovine brucellosis, Brucella abortus strain 19, buffered acidified plate agglutination antigen.

$$
\text { (http://www.bvmj.bu.edu.eg) BVMJ-36(1): 43-50, 2019) }
$$

\section{INTRODUCTION}

Brucellosis is a worldwide zoonotic infectious disease caused by Gram-negative bacteria from the genus Brucella. Animal brucellosis has been recorded in Egypt since 1939 and the prevalence of serological reactors on limited surveys has varied from one survey to another with a range between $16.5 \%$ to $23 \%$ in cattle and $7 \%$ to $10 \%$ in buffaloes. In 2002, the prevalence of positive serological reactors was $3 \%$ in cattle and $2 \%$ in buffaloes. Diagnosis of Brucellosis depends on bacteriological isolation from abortion material, udder secretions or tissues collected at post-mortem. Presumptive diagnosis can be made by assessing specific cell-mediated or serological responses to Brucella antigens (OIE, 2012). Among the rapid agglutination assays for brucellosis surveillance are the Buffered Acidified Plate antigen (BAPA) and the Rose -Bengal plate tests. The Rose -Bengal or brucellosis card test $(\mathrm{BCT})$ is rapid qualitative one dilution plate agglutination at acidic $\mathrm{pH}$ of $3.65 \pm 0.05$ attained by lactate buffered phenol saline in which inactivated Rose -Bengal stained Brucella abortus cells are suspended and 
standardized. The test brings about agglutination of the non-agglutinogenic $\mathrm{IgG}_{1}$ distinctive of the longstanding Brucella infection (Alton etal., 1988). This adds up for more sensitivity and specificity to the test.It was used after the presumptive BAPA test, the Rose - Bengal plate test (RBPT) reduces the number of positive samples demanding confirmation. All of these tests are recommended for international trade in the (OIE Terrestrial Manual 2016). It is prospective that smooth Brucella abortus strain 19 (S-19) as a vaccinal strain, could be used as substitute for S.99 in to preparation of antigen.

The present work aimed to evaluate the specificity and sensitivity of buffered acidified plate agglutination antigen prepared from B. abortus S19 against corresponding antigens traditionally prepared from strain 99 used for diagnosis of bovine brucellosis in cows of non-vaccinated history and its relation to the sensitivity of Rose Bengal test.

\section{MATERIALS AND METHODS}

\subsection{Brucella strains:}

Smooth Brucella abortus biovar 1 strain 99(S-99) (Weybridge, England) and smooth Brucella abortus biovar 1 strain 19(S-19)(CZ Veterinaria, S.A., Spain) were used.

\subsection{Brucella antigens for serologic tests:}

\subsubsection{Conventional Rose Bengal and Buffer} Acidified Plate antigens (BAPA) prepared from B.abortus (S-99) were supplied by Veterinary Serum and Vaccine Research Institute, Abbasia, Cairo (VSVRI). It prepared according to international rules regarding $\mathrm{pH}$ and PCV (Alton et al., 1988 and OIE, 2016).

\subsubsection{Buffer Acidified Plate antigen (BAPA) prepared from $B$. abortus}

Strain 19 (S-19) was locally prepared according to Alton et al., (1988).

\subsection{Serum samples:}

A total 4100 serum samples were collected from non-vaccinated cows against Brucellosis from five dairy farms in different governorates in Egypt as list in details in (Table 1). History of the farms reveled of these farms were of no history of vaccination against brucellosis. All examined animals did not show abortion or retained placenta. Animals which have positive results of serological tests were slaughtered immediately, in each farm sera were tested 27 times with various intervals as shown in (Table 2 to 6 ).

\subsection{Serologic tests (Alton et al. 1988)}

Rose Bengal (RB) test was performed, following the procedure described by Alton et al. (1988), through mixing of $25 \mathrm{ul}$ of sera and $25 \mathrm{ul}$ of the antigen. The plates were shaken for 4 min and any agglutination that appeared within this time was recorded as a positive reaction.

BAPA test was carried out, mixing $80 \mathrm{ul}$ of sera and $30 \mathrm{ul}$ of the antigen. The plates were shaken for $8 \mathrm{~min}$ and any agglutination that appeared within this time was recorded as a positive reaction

\section{RESULTS}

Results are summarized in Tables (3) to (11) and Figures (1) to (4).Tables (4) to (8) reveal the overall performance of all acidified plate agglutination test (conventional RBA, conventional BAPA and S.19 BAPA) in each of five farms of cattle. The performance characteristics included the positive and negative result of each agglutination tests, numbers of these tests, numbers of samples in each one and Length of time interval between each test.It respect of rose Bengal test, sensitivity and specificity of the two BAPA antigens preparations were calculated on (http://vassarstats.net/clin1.html) as shown in table (3). 
Lubna, Farahat et al., (2019). BVMJ-36 (1): $43-50$

Table (1): Epidemiologic data of dairy farms included in the current study

\begin{tabular}{|c|c|c|c|c|c|}
\hline \multirow{2}{*}{ Farms } & \multicolumn{2}{|c|}{ Animals } & \multirow[t]{2}{*}{ Governorate } & \multirow{2}{*}{$\begin{array}{l}\text { Numbers of } \\
\text { sera collected }\end{array}$} & \multirow{2}{*}{$\begin{array}{c}\text { Management } \\
\text { Nutration } \\
\text { Biosafety level }\end{array}$} \\
\hline & Breed & Age & & & \\
\hline First & Dairy & 36 month & Beni-Seuf & 1000 & Medium \\
\hline Second & Dairy & 36 month & El-Beheira & 600 & Good \\
\hline Third & Holstein Friesian & 2 years & Alexandria Desert Road & 1800 & Very Good \\
\hline Fourth & Dairy & 36 month & Wadi El Natrun & 350 & Good \\
\hline Fifth & Dairy & 36 month & Hosh Essa El-Beheira & 350 & Medium \\
\hline Total & & & & 4100 & \\
\hline
\end{tabular}

Table (2): Total number of tested serum samples collected from cows in different farms in Egypt.

\begin{tabular}{|c|c|c|c|c|}
\hline Farms & $\begin{array}{c}\text { Total Numbers } \\
\text { of animals }\end{array}$ & $\begin{array}{c}\text { No.of Serological } \\
\text { Tests }\end{array}$ & $\begin{array}{l}\text { No. of Samples } \\
\text { in Every Tests }\end{array}$ & $\begin{array}{c}\text { Time Interval } \\
\text { Between Every Test }\end{array}$ \\
\hline 1st farm & 1000 & 6 tests & $\begin{array}{l}\text { 1st test (1000)samples } \\
\text { 2nd test (976) samples } \\
\text { 3rd test (967) samples } \\
\text { 4th test (961) samples } \\
\text { 5th test (952) samples } \\
\text { 6thtest (945) samples }\end{array}$ & $\begin{array}{l}\text { Every } \\
21 \\
\text { days }\end{array}$ \\
\hline 2nd farm & 600 & 6 tests & $\begin{array}{l}\text { 1st test (600)samples } \\
\text { 2nd test (599) samples } \\
\text { 3rd test (599) samples } \\
\text { 4th test (599) samples } \\
\text { 5th test ( 597) samples } \\
\text { 6thtest (597) samples }\end{array}$ & $\begin{array}{l}\text { Most of tests apply every } \\
3 \text { months except the period } \\
\text { interval between the } 3 \mathrm{rd} \\
\text { and } 4 \text { th is } 6 \text { months. }\end{array}$ \\
\hline 3rd farm & 1800 & 7 tests & $\begin{array}{l}1 \text { st test } 1800 \\
2^{\text {nd }} \text { test } 1785 \\
3^{\text {rd }} \text { test } 1775 \\
4^{\text {th }} \text { test } 1770 \\
5^{\text {th }} \text { test } 1767 \\
6^{\text {th }} \text { test } 1763 \\
7^{\text {th }} \text { test } 1761\end{array}$ & $\begin{array}{c}21 \\
\text { days Interval Between } \\
\text { Every Test }\end{array}$ \\
\hline 4th farm & 350 & 2 tests & $\begin{array}{l}\text { 1st test 350samples } \\
2 \text { nd test 350samples }\end{array}$ & $\begin{array}{l}3 \text { months interval } \\
\text { between } \\
2 \text { tests }\end{array}$ \\
\hline 5 th farm & 350 & 3 tests & $\begin{array}{l}1 \text { st test } 350 \text { samples } \\
2^{\text {nd }} \text { test } 348 \text { samples } \\
3^{\text {rd }} \text { test } 347 \text { samples }\end{array}$ & $\begin{array}{c}3 \text { months interval between } \\
\text { every tests }\end{array}$ \\
\hline Total & 4100 & 24 tests & & \\
\hline
\end{tabular}

Table (3): Calculation of sensitivity and specificity with respect of gold standard test.

\begin{tabular}{lcccc}
\hline & & \multicolumn{2}{c}{ Gold standard test (cft) } & Total \\
& & Positive & Negative & \\
\hline Test under evaluation & Positive & $\mathrm{A}$ & $\mathrm{B}$ & $\mathrm{A}+\mathrm{b}$ \\
& Negative & $\mathrm{C}$ & $\mathrm{D}$ & $\mathrm{C}+\mathrm{d}$ \\
Total & & $\mathrm{A}+\mathrm{c}$ & $\mathrm{B}+\mathrm{d}$ & $\mathrm{N}(264)$ \\
\hline Sensitivity $=$ total positive/ total samples & \multicolumn{2}{c}{ Relative Sensitivity $=\mathrm{A} / \mathrm{A}+\mathrm{C}$} \\
Specificity $=\mathrm{D} / \mathrm{D}+\mathrm{B}$ & & True positive (Positive Predictive Value) $=\mathrm{A} / \mathrm{A}+\mathrm{B}$ \\
False positive $=\mathrm{A} / \mathrm{A}+\mathrm{B}$ & & True negative (Negative Predictive Value) $=\mathrm{D} / \mathrm{C}+\mathrm{D}$ \\
False negative $=\mathrm{C} / \mathrm{C}+\mathrm{D}$ & & &
\end{tabular}


3.1 Sero-diagnostic efficacy of antigens (conventional RBA, conventional BAPA and
S.19 BAPA) prepared from B.abortus biovar 1 (S.99, S.19) used for agglutination tests.

Table (4): Performance of the conventional Rose Bengal test, (S.99) BAPA, (S.19) BAPA in the first farm.

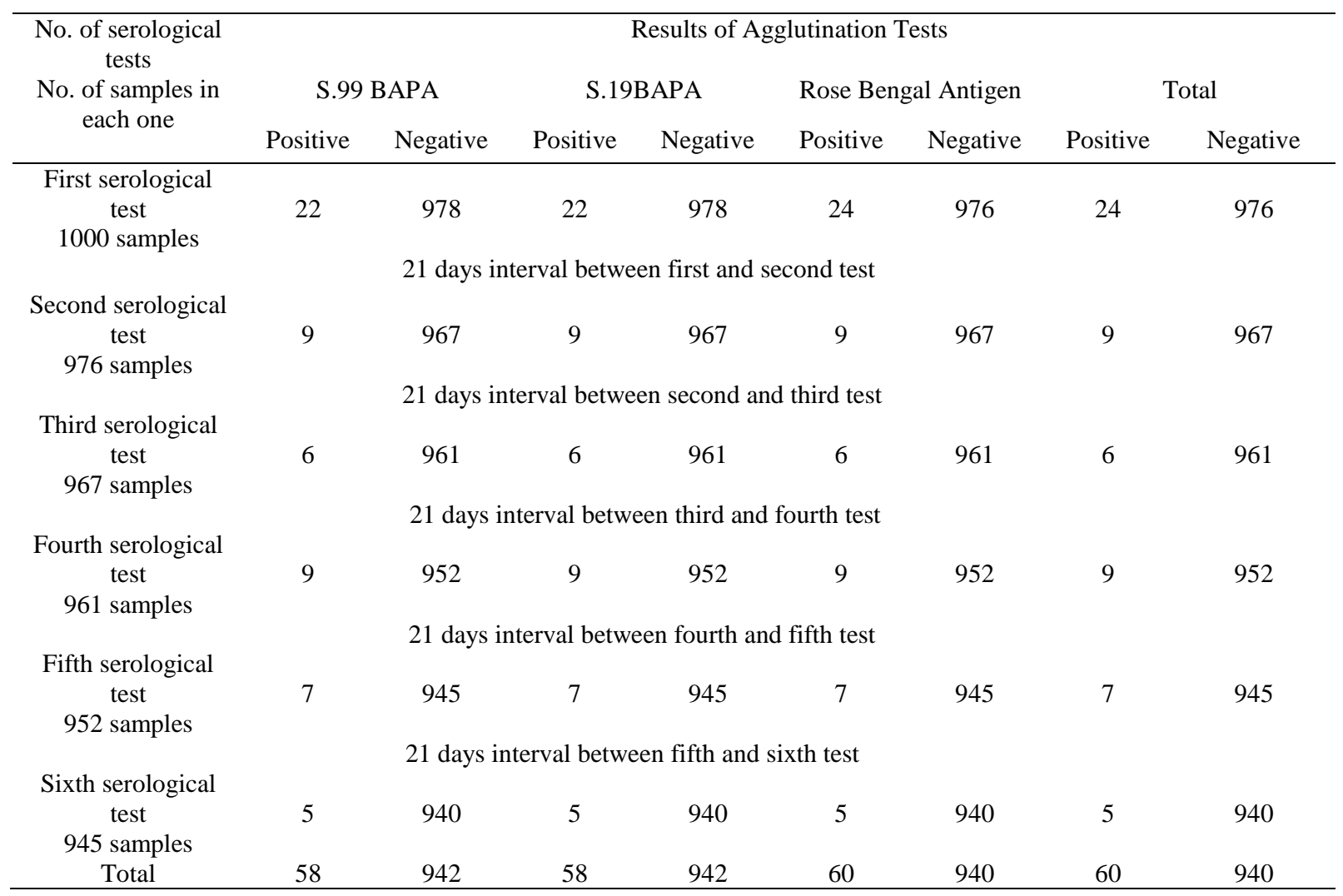

Table (5): Performance of the conventional Rose Bengal test, (S.99) BAPA, (S.19) BAPA in the second farm.

\begin{tabular}{|c|c|c|c|c|c|c|c|c|}
\hline \multirow{3}{*}{$\begin{array}{l}\text { No. of serological tests } \\
\text { No. of samples in each } \\
\text { one }\end{array}$} & \multicolumn{8}{|c|}{ Results of Agglutination Tests } \\
\hline & \multicolumn{2}{|c|}{ S.99 BAPA } & \multicolumn{2}{|c|}{ S.19BAPA } & \multicolumn{2}{|c|}{ Rose Bengal Antigen } & \multicolumn{2}{|c|}{ Total } \\
\hline & Positive & Negative & Positive & Negative & Positive & Negative & Positive & Negative \\
\hline $\begin{array}{l}\text { First serological test } \\
600 \text { samples }\end{array}$ & 1 & 599 & 1 & 599 & 1 & 599 & 1 & 599 \\
\hline & 3 months in & rval betwe & $n$ first and & econd test & & & & \\
\hline $\begin{array}{l}\text { Second serological test } \\
599 \text { samples }\end{array}$ & 0 & 599 & 0 & 599 & 0 & 599 & 0 & 599 \\
\hline $\begin{array}{l}\text { Third serological test } \\
599 \text { samples }\end{array}$ & $\begin{array}{c}0 \\
6 m o n t h s i n\end{array}$ & 599 & second a & $\begin{array}{l}d \text { third test } \\
599 \\
\end{array}$ & 0 & 599 & 0 & 599 \\
\hline $\begin{array}{l}\text { Fourth serological test } \\
599 \text { samples }\end{array}$ & $\begin{array}{c}2 \\
3 \text { months in }\end{array}$ & 597 & $\begin{array}{c}2 \\
\text { fourth }\end{array}$ & 597 & 2 & 597 & 2 & 597 \\
\hline $\begin{array}{l}\text { Fifth serological test } \\
597 \text { samples }\end{array}$ & 0 . & 597 & 0 & 597 & 0 & 597 & 0 & 597 \\
\hline $\begin{array}{l}\text { Sixth serological test } \\
597 \text { samples } \\
\text { Total }\end{array}$ & 3months il & erval betw & en fifth an & sixth test & $\begin{array}{l}0 \\
3\end{array}$ & $\begin{array}{l}597 \\
597\end{array}$ & $\begin{array}{l}0 \\
3\end{array}$ & $\begin{array}{l}597 \\
597\end{array}$ \\
\hline
\end{tabular}


Lubna, Farahat et al., (2019). BVMJ-36 (1): $43-50$

Table (6): Performance of the conventional Rose Bengal test, (S.99) BAPA, (S.19) BAPA in the third farm.

\begin{tabular}{|c|c|c|c|c|c|c|c|c|}
\hline \multirow{3}{*}{$\begin{array}{c}\text { No. of serological tests } \\
\text { No.of samples in each } \\
\text { one }\end{array}$} & \multicolumn{8}{|c|}{ Results of Agglutination Tests } \\
\hline & \multicolumn{2}{|c|}{ S.99 BAPA } & \multicolumn{2}{|c|}{ S.19BAPA } & \multicolumn{2}{|c|}{ Rose Bengal Antigen } & \multicolumn{2}{|c|}{ Total } \\
\hline & Positive & Negative & Positive & Negative & Positive & Negative & Positive & Negative \\
\hline $\begin{array}{c}\text { First serological test } \\
1800 \text { samples }\end{array}$ & 15 & 1785 & 15 & 1785 & 15 & 1785 & 15 & 1785 \\
\hline \multicolumn{9}{|c|}{21 days interval between first and second test } \\
\hline $\begin{array}{l}\text { Second serological test } \\
1785 \text { samples }\end{array}$ & 9 & $\begin{array}{l}1776 \\
\text { days interve }\end{array}$ & $\begin{array}{l}9 \\
\text { between se }\end{array}$ & $\begin{array}{l}1776 \\
\text { nd and thir }\end{array}$ & $\begin{array}{l}10 \\
\text { est }\end{array}$ & 1775 & 10 & 1775 \\
\hline $\begin{array}{l}\text { Third serological test } \\
1775 \text { samples }\end{array}$ & 5 & $\begin{array}{l}1770 \\
\text { days interva }\end{array}$ & $\begin{array}{l}5 \\
\text { between th }\end{array}$ & $\begin{array}{l}1770 \\
\text { and fourth }\end{array}$ & $\begin{array}{c}5 \\
\text { st }\end{array}$ & 1770 & 5 & 1770 \\
\hline $\begin{array}{l}\text { Fourth serological test } \\
1770 \text { samples }\end{array}$ & 3 & $\begin{array}{l}1767 \\
\text { divs interva }\end{array}$ & 3 & 1767 & 3 & 1767 & 3 & 1767 \\
\hline $\begin{array}{l}\text { Fifth serological test } \\
1767 \text { samples }\end{array}$ & 3 & $\begin{array}{l}1764 \\
\text { days interva }\end{array}$ & $\begin{array}{l}3 \\
\text { oetween fif }\end{array}$ & $\begin{array}{l}1764 \\
\text { and sixth te }\end{array}$ & 4 & 1763 & 4 & 1763 \\
\hline $\begin{array}{c}\text { Sixth serological test } \\
1763 \text { samples }\end{array}$ & 2 & 1761 & 2 & 1761 & 2 & 1761 & 2 & 1761 \\
\hline $\begin{array}{l}\text { Seventh serological test } \\
1761 \text { samples }\end{array}$ & 1 & 1760 & 1 & 1760 & 1 & 1760 & 1 & 1760 \\
\hline Total & 38 & 1762 & 38 & 1762 & 40 & 1760 & 40 & 1760 \\
\hline
\end{tabular}

Table (7): Performance of the conventional Rose Bengal test, (S.99) BAPA, (S.19) BAPA in the fourth farm.

\begin{tabular}{|c|c|c|c|c|c|c|c|c|}
\hline \multirow{3}{*}{$\begin{array}{c}\text { No. of serological tests } \\
\text { No.of samples in each } \\
\text { one }\end{array}$} & \multicolumn{8}{|c|}{ Results of Agglutination Tests } \\
\hline & \multicolumn{2}{|c|}{ S.99 BAPA } & \multicolumn{2}{|c|}{ S.19BAPA } & \multicolumn{2}{|c|}{ Rose Bengal Antigen } & \multicolumn{2}{|c|}{ Total } \\
\hline & Positive & Negative & Positive & Negative & Positive & Negative & Positive & Negative \\
\hline $\begin{array}{l}\text { First serological test } \\
350 \text { samples }\end{array}$ & 0 & 350 & 0 & 350 & 0 & 350 & 0 & 350 \\
\hline $\begin{array}{l}\text { Second serological test } \\
350 \text { samples } \\
\text { Total }\end{array}$ & $\begin{array}{l}0 \\
0\end{array}$ & lonths inter & between $\mathrm{f}$ & $t$ and secon & $\begin{array}{l}0 \\
0\end{array}$ & $\begin{array}{l}350 \\
350\end{array}$ & $\begin{array}{l}0 \\
0\end{array}$ & $\begin{array}{l}350 \\
350\end{array}$ \\
\hline
\end{tabular}

Table (8): Performance of the conventional Rose Bengal test, (S.99) BAPA, (S.19) BAPA in the fifth farm.

\begin{tabular}{|c|c|c|c|c|c|c|c|c|}
\hline \multirow{3}{*}{$\begin{array}{l}\text { No. of serological tests } \\
\text { No.of samples in each one }\end{array}$} & \multicolumn{8}{|c|}{ Results of Agglutination Tests } \\
\hline & \multicolumn{2}{|c|}{ S.99 BAPA } & \multicolumn{2}{|c|}{ S.19BAPA } & \multicolumn{2}{|c|}{ Rose Bengal Antigen } & \multicolumn{2}{|c|}{ Total } \\
\hline & Positive & Negative & Positive & Negative & Positive & Negative & Positive & Negative \\
\hline \multicolumn{9}{|l|}{ First serological test } \\
\hline 350 samples & 2 & 348 & 2 & 348 & 2 & 348 & 2 & 348 \\
\hline \multicolumn{9}{|c|}{3 months interval between first and second test } \\
\hline Second serological test & & & & & & & & \\
\hline 348 samples & 1 & 347 & 1 & 347 & 1 & 347 & 1 & 347 \\
\hline \multicolumn{9}{|c|}{3 months interval between second and third test } \\
\hline $\begin{array}{l}\text { Third serological test } \\
347 \text { samples }\end{array}$ & 0 & 347 & 0 & 347 & 0 & 347 & 0 & 347 \\
\hline Total & 3 & 347 & 3 & 347 & 3 & 347 & 3 & 347 \\
\hline
\end{tabular}




\section{Preparation of buffered acidified plate antigen from Brucella abortus strain 19}

Table (9): Comparison between the diagnostic importance of strain 19 BAPA and conventional antigens (Rose Bengal Antigen and strain 99 BAPA) by using 4100 serum samples from naturally infected cattle in different farms.

\begin{tabular}{|c|c|c|c|c|c|c|}
\hline \multirow{4}{*}{$\begin{array}{c}\text { Diagnostic Tests } \\
\text { Strains } \\
\text { Agglutination } \\
\text { Tests }\end{array}$} & \multicolumn{6}{|c|}{ Plate Agglutination Tests using antigens prepared from } \\
\hline & \multicolumn{4}{|c|}{ Strain 99} & \multicolumn{2}{|c|}{ Strain 19} \\
\hline & \multicolumn{2}{|c|}{ RBT } & \multicolumn{2}{|c|}{ BAPA } & \multicolumn{2}{|c|}{ BAPA } \\
\hline & $\begin{array}{l}\text { Numbers of } \\
\text { positive } \\
\text { reactor }\end{array}$ & $\begin{array}{l}\text { Numbers of } \\
\text { negative } \\
\text { reactor }\end{array}$ & $\begin{array}{l}\text { Numbers of } \\
\text { positive } \\
\text { reactor }\end{array}$ & $\begin{array}{c}\text { Numbers of } \\
\text { negative } \\
\text { reactor }\end{array}$ & $\begin{array}{l}\text { Numbers of } \\
\text { positive } \\
\text { reactor }\end{array}$ & $\begin{array}{c}\text { Numbers of } \\
\text { negative } \\
\text { reactor }\end{array}$ \\
\hline First Farm & 60 & 940 & 58 & 942 & 58 & 942 \\
\hline Second Farm & 3 & 597 & 3 & 597 & 3 & 597 \\
\hline Third Farm & 40 & 1760 & 38 & 1762 & 38 & 1762 \\
\hline Fourth Farm & 0 & 350 & 0 & 350 & 0 & 350 \\
\hline Fifth Farm & 3 & 347 & 3 & 347 & 3 & 347 \\
\hline Total & 106 & 3994 & 102 & 3998 & 102 & 3998 \\
\hline
\end{tabular}

Table (10): Total Positivity Percent in all Farms of all plate agglutination tests.

\begin{tabular}{|c|c|c|c|c|c|c|}
\hline \multirow{4}{*}{$\begin{array}{c}\text { Diagnostic Tests } \\
\text { Strains } \\
\text { Agglutination } \\
\text { Tests } \\
\text { Total percent of } \\
\text { Positivity and } \\
\text { Negativity }\end{array}$} & \multicolumn{6}{|c|}{ Plate Agglutination Tests using antigens prepared from } \\
\hline & \multicolumn{4}{|c|}{ Strain 99} & \multicolumn{2}{|c|}{ Strain 19} \\
\hline & \multicolumn{2}{|c|}{ RBT } & \multicolumn{2}{|c|}{ BAPA } & \multicolumn{2}{|c|}{ BAPA } \\
\hline & Positivity $\%$ & $\begin{array}{c}\text { Negativity } \\
\%\end{array}$ & Positivity \% & $\begin{array}{c}\text { Negativity } \\
\%\end{array}$ & Positivity $\%$ & $\begin{array}{c}\text { Negativity } \\
\%\end{array}$ \\
\hline First Farm & $6 \%$ & $94 \%$ & $5,8 \%$ & $94,2 \%$ & $5,8 \%$ & $94,2 \%$ \\
\hline Second Farm & $0,5 \%$ & $99,5 \%$ & $0,5 \%$ & $99,5 \%$ & $0,5 \%$ & $99,5 \%$ \\
\hline Third Farm & $2,22 \%$ & $97,77 \%$ & $2,1 \%$ & $97,88 \%$ & $2,1 \%$ & $97,88 \%$ \\
\hline Fourth Farm & $0 \%$ & $100 \%$ & $0 \%$ & $100 \%$ & $0 \%$ & $100 \%$ \\
\hline Fifth Farm & $0,85 \%$ & $99,14 \%$ & $0,857 \%$ & $99,14 \%$ & $0,857 \%$ & $99,14 \%$ \\
\hline Total & $2,6 \%$ & $97,41 \%$ & $2,5 \%$ & $97,51 \% \%$ & $2,5 \%$ & $97,51 \%$ \\
\hline
\end{tabular}

Table (11): Results of BABA tests against Rose Bengal test as a Gold Standard test.

\begin{tabular}{lccc}
\hline Tests & Antigens & $\begin{array}{c}\text { Rose Bengal test } \\
\text { +ve }\end{array}$ & -ve \\
\hline $\begin{array}{l}\text { Buffered acidified plate } \\
\text { agglutination test }\end{array}$ & Buffered acidified & 102 & 0 \\
\hline
\end{tabular}




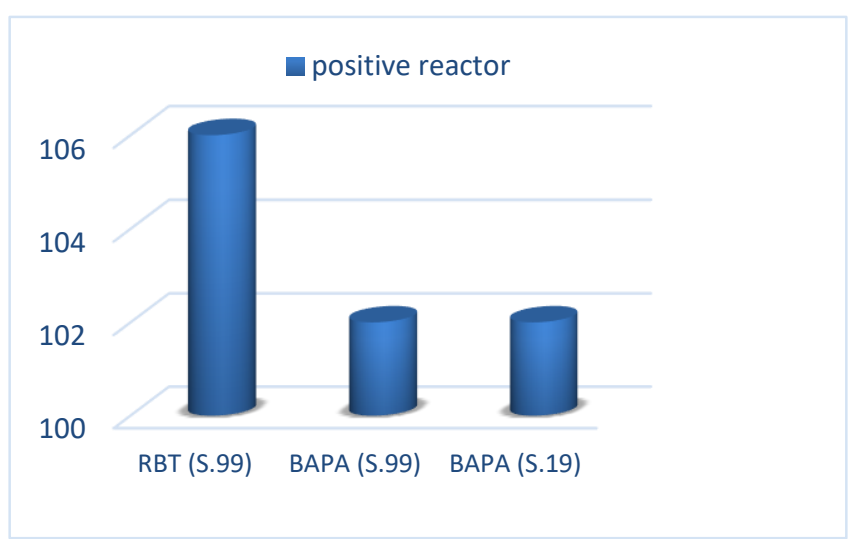

Figure (1): Total positive reactor of Plate Agglutination Tests in all farms.

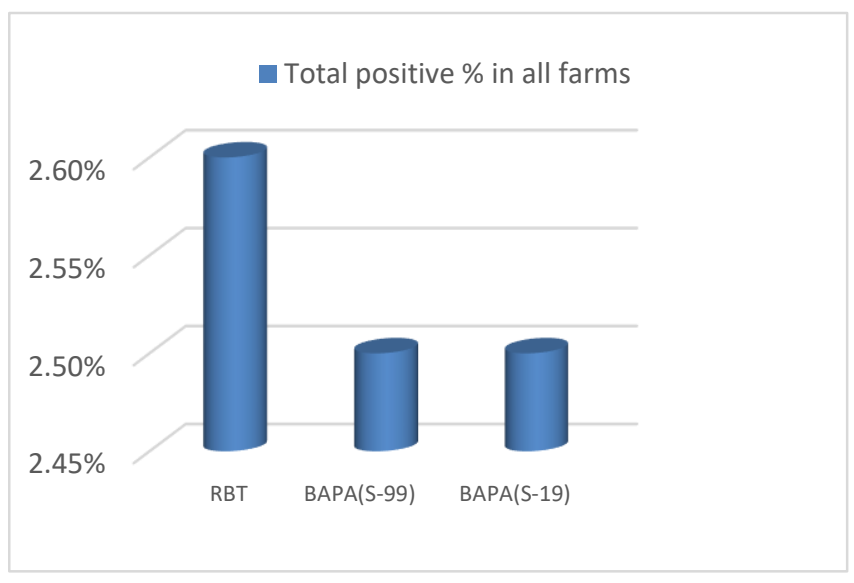

Figure (3): Total positivity percent of Plate Agglutination Tests in all farms

\section{DISCUSION}

The Rose Bengal plate agglutination, Buffered Acidified Plate Agglutination, complement fixation and indirect ELISA tests are usually recommended for screening flocks and individual animals for brucellosis. The complement fixation test is the only test prescribed for confirmation and international trade, but other tests as the Agar Gel Perception test, immunodiffusion and competitive ELISA, are useful for confirmation purposes. All over the world, all agglutination tests use the $B$. abortus strain 99 or 1119 antigens although in some cases different strains were used Erganis et al. (2005) used Brucella melitensis and Brucella suis S2 antigens. The Buffered Antigen Plate

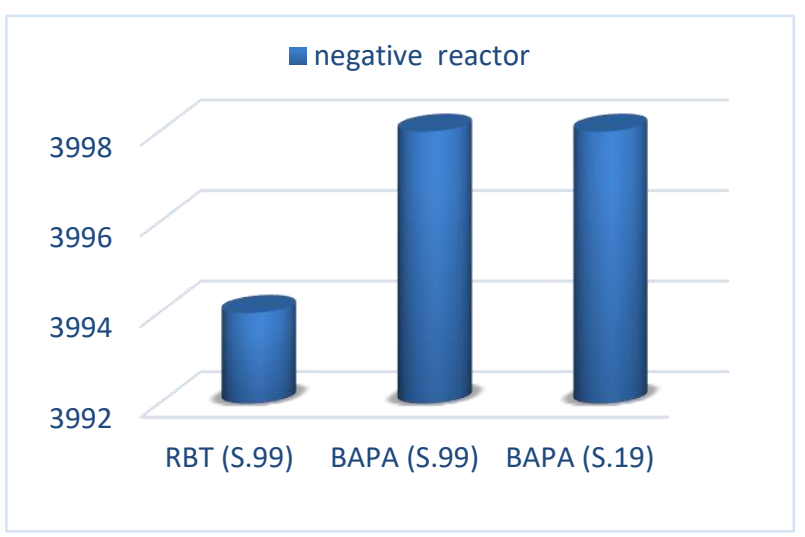

Figure (2): Total negative reactor of Plate Agglutination Tests in all farms.

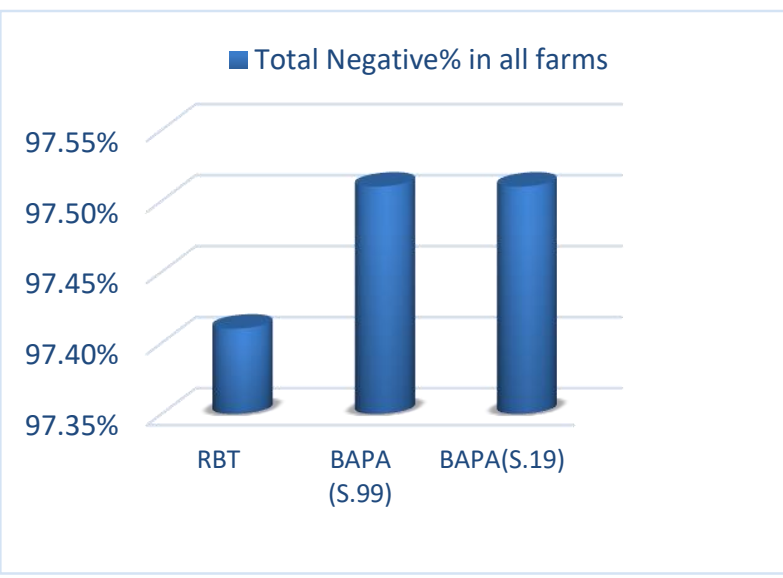

Figure (4): Total Negativity percent of Plate Agglutination Tests in all farms.

Agglutination test (BPAT) has been widely used (Angus and Barton, 1984 and Nielsen and $\mathrm{Yu}, 2010)$ and also the Rose Bengal test (RBT) (Nielsen and Yu, 2010 and Morgan etal., 1969).

Among the rapid agglutination assays for brucellosis surveillance and rapid field diagnosis are the Buffered Acidified Plate antigen (BAPA) and the Rose -Bengal plate tests. Both tests are well known as a pilot (screening), cheap, effective and rapid test for the diagnosis of brucellosis. It can be performed with the minimum of facilities, and the end result is read by the naked eye. Because of its apparent simplicity, high level of standardization of antigen and accuracy of reading is needed (Erganis et al., 2005). 
After the presumptive diagnosis by BAPA test, using the Rose -Bengal plate test (RBPT) reduced the number of positive samples demanding confirmation. Each of these tests is recommended for international trade (OIE, 2016). It is prospective that smooth Brucella abortus (S-19) as a vaccinal strain, could be used as a substitute for S.99 in order to prepare Brucella antigens (Alton et al., 1988).

In this study 4100 bovine serum were tested against all prepared rapid slide agglutination antigens. Rose Bengal test prepared from $B$. abortus S99 was considered as a gold standard test to determine the sensitivity and specificity of tested BAPA antigens in absence of bacteriological isolation. Statistics in this study was considered the $95 \%$ confidence intervals. In this study, No satisfactory differences were observed in specificity and sensitivity of tested BAPA antigens prepared from different brucella reference strains with constant $\mathrm{PCV}$ and $\mathrm{pH}$. With respect to Rose Bengal test and according to results in table(10)and figure(1), sensitivity of different antigens preparations were calculated on (http://vassarstats.net/clin1.html)

with 95\% Confidence Intervals (CI) as shown in (table3). Sensitivity of the Rose Bengal test was $2.59 \%$ where the sensitivity of the slide agglutination test using the two tested BAPA antigens was equal to each other (2.49\%). Relative sensitivity, specificity, true positive and truce negative of both antigens were $96.2 \%, 50 \%, 2.49 \%$ and $97.51 \%$ respectively and prevalence of the diseases in tested farms were $1.31 \%$. The results, in this study revealed that there are no significant differences between conventional antigens and these prepared from brucella abortus biovar 1 strain 19 as recommended by Alton et al., (1988).

\section{CONCLUSION}

According to the diagnostic performance parameters obtained under conditions of this study, It is concluded that the Buffer Acidified Plate antigen (BAPA) prepared from Brucella abortus biovar 1 strain $19(\mathrm{~S}$ -
19) gave similar results to that antigen prepared from Brucella abortus (Weybridge) (S99) on sera collected from the naturally infected farms . So, Brucella abortus strain19 (S-19) and Brucella abortus strain 99 are indistinguishable for the preparation of Brucella antigens. So, Brucella abortus strain19 (S-19) could be used as replacement of Brucella abortus strain 99 to prepare the Buffer Acidified Plate antigen (BAPA) for large scale production of such antigens in Egypt.

\section{REFERANCES}

Alton, G.G.; Jones, L.M.; Angus, R.D.; Verger, J.M.((1988):Techniques for the Brucellosis Laboratory; Instituttional de la Recherche Agronomique: Paris, France, 1988; pp. $17-62$.

Angus, R. D.; \& Barton, C. E.; (1984). The production and evaluation of a buffered plate antigen for use in a presumptive test for brucellosis. Developments in biological Standardization, 56, 349-356.

Erganis, H.H. ; Hadimli, H. ; Solmaz, (2005) : Brucella Melitensis and Brucella Suis

Morgan, D.J. ;Mackinnon, J.R. ; Lawson, (1969) The rose bengal plate agglutination test in the diagnosis of brucellosis.

Nielsen, K.; Yu. WL. (2010): Serological Diagnosis of Brucellosis.

OIE: (2012): Seroprevalence of brucellosis in sheep and isolation of Brucella abortus biovar 6 in Kassala State Eastern Sudan.

OIE:(2016). Chapter 2.1.4. Manual of Diagnostic Tests and Vaccines for Terrestrial Animals. Office International des Epizooties, Paris 99 